\title{
Erkennen, behandeln und verhüten von Depressionen in der Grundversorgung
}

\author{
Die Behandlung depressiver Erkrankungen in der hausärztlichen Praxis ist meist ein- \\ fach und kostengünstig. Daher würde sich die Förderung der Effizienz durch Weiter- \\ und Fortbildungsmassnahmen lohnen. Versuche zur Optimierung von Diagnostik, \\ Therapie und Prävention existieren. Ein Kompetenzprofil als Orientierungshilfe für \\ die hausärztliche Praxis wäre sinnvoll.
}

Stefan Begréa,

Regula Rička ${ }^{b}$

a Dr. med., FMH Innere Medizin, Spez. Psychosomatik FAPPM, FMH Psychiatrie und Psychotherapie, Leiter Weiterbildungsstätte Psychiatrie C. L. Lory-Haus, Chefarzt-Stv. Psychosomatik, Inselspital Bern, Präsident WK Schweizerische Akademie für Psychosomatische und Psychosoziale Medizin SAPPM, zugezogener externer Berater

b PhD, MPH, Bundesamt für Gesundheit

Wir danken Hans Kurt von der SGPP und FMPP, Pierre Loeb von der SAPPM, Ueli Grüninger vom KHM sowie Elisabeth Bandi vom IHAM Zürich und Brigitte Fahrländer, Hausärztin in Schüpfen, BE, für das kritische Gegenlesen.

Korrespondenz:

Dr. med. Stefan Begré

Klinik für

Allgemeine Innere Medizin

Inselspital

CH-3010 Bern

Tel. 0316322019

Fax 0313821184

stefan.begre@insel.ch

www.inneremedizin.insel.ch

\section{Einleitung}

Weltweit ist Depression die häufigste Ursache für Erwerbsunfähigkeit [1]. Durch keine andere Erkrankung gehen in den Industrieländern mehr gesunde Lebensjahre verloren als durch Depressionen [2]. Depression wird zunehmend als chronische, rezidivierende Krankheit eingeschätzt [3]. Depression ist die häufigste Ursache für Suizid [4]. 50\% der Depressiven sind suizidal während einer depressiven Episode und 10-15\% der Depressiven suizidieren sich. 90\% der Suizidanten litten unter einer Depression [1]. Aus diesem Grund lancierte die WHO weltweit verschiedene Informationskampagnen, die zu vermehrten Aktivitäten zur Verbesserung der Prävention, Diagnostik und Behandlung von Depressionen in den verschiedenen Ländern führten.

\section{Internationale und nationale evidenzbasierte Aktionen}

Bereits in den 90er Jahren hat die FMH mit Unterstützung durch das Bundesamt für Gesundheit, Interpharma, die Schweizerische Vereinigung privater Kranken- und Unfallversicherer sowie durch die Schweizerische Stiftung für Gesundheitsförderung ein Konzept zur Krisenbewältigung und Suizidverhütung entwickelt. Im Mittelpunkt des damaligen Konzepts standen die Weiter- und Fortbildung für die Ärztinnen und Ärzte sowie Seminare für Medienschaffende [5]. Die Initiative der FMH stützte sich auf die Interventionsstudie der Insel Gotland von 1983/84. Rutz konnte mit seinen Mitarbeitenden damals wissenschaftlich nachweisen, dass das intensive Fortbildungsprogramm über die Diagnostik und Behandlung depressiver Störungen zur Senkung der Suizidrate führte [6].

2000-2002 etablierte Hegerl (2006) in einer Interventionsstudie in Nürnberg das gemeindeorientierte Programm zur Früherkennung und Behandlungsoptimierung der Depression «Bündnis gegen Depression». Dieser Mehrebenenansatz umfasst gezielte Fortbildungen für die Ärzteschaft in der hausärztlichen Praxis, die Pflegenden und weiteren wichtigen Multiplikatoren, kombiniert mit einer Informationskampagne für die

\section{Reconnaître, soigner et prévenir les dépressions dans la médecine de premiers recours}

La dépression et le suicide sont des phénomènes très fréquents en Suisse. Le diagnostic et le traitement des maladies dépressives puissent être effectués la plupart du temps facilement et à moindres coûts par des médecins généralistes. Et de ce fait il serait profitable de consolider la mise en pratique de cet évidence par une éducation et formation professionnelle continue finement ciblée. Le présent article souligne cette difficulté, décrit les solutions proposées jusqu'à présent aux niveaux national et international pour optimiser le diagnostic, le traitement et la prévention et propose un profil de compétences d'orientation à l'intention des médecins généralistes. II invite d'établir un catalogue correspondant, qui sera présenté de manière plus détaillée dans un article suivant du Bulletin des médecins suisses (BMS). Cette mesure a été prise car la Suisse dispose, tant sur le plan national que cantonal, d'un système de formation de base, postgrade et continue très bien établi et organisé. Ainsi, une collaboration avec les organisations spécialisées des médecins, des psychologues et du personnel soignant permet de trouver des solutions de longue durée pour distinguer et prendre en charge des personnes à tendance dépressive et suicidaire.

Bevölkerung und einem Angebot zur Selbsthilfe. Mit der Informationskampagne soll die Bevölkerung sachlich über die Depression informiert werden und Vorurteile gegenüber der Depression und der Behandlung 
sollen abgebaut werden. Gegenüber der Kontrollregion führte dieses Programm in der Stadt Nürnberg zu einer positiven Bilanz. Die Suizidrate in Nürnberg sank nach zwei Jahren um $25 \%$. Im selben Zeitraum gingen die Suizidversuche um 26\% zurück.

Das erprobte Programm erlangte eine rasche Verbreitung innerhalb von Deutschland. Im Rahmen des Europäischen Public-Health-Programms führten 18 weitere EU-Mitgliedsstaaten breitgefächerte gemeindebasierte Interventionsprogramme ein. In den Jahren 2003-2005 erprobte der Kanton Zug das Programm in Zusammenarbeit mit dem Bundesamt für Gesundheit (BAG) und der Selbsthilfeorganisation Equilibrium erstmals für die Schweiz. 2004 startete ein weiteres Projekt im Kanton Bern. Im Jahr 2006 hat das BAG die Nutzungsrechte in Deutschland für die gesamte Schweiz erworben und gibt diese kostenlos mit einer Vereinbarung an interessierte Kantone weiter. Seither haben bereits fünf Kantone dieses Angebot genutzt. Zur Weiterentwicklung des Konzepts im schweizerischen Kontext und für eine nachhaltige Verankerung in den schweizerischen Strukturen unterstützt das BAG zurzeit die folgenden Projekte:

- Finanzielle Unterstützung für das Monitoring Suizidales Verhalten in der Agglomeration Bern seit 2004.

- Erhebung der Depression im Sentinella-Meldesystem 2008/09 in Zusammenarbeit mit deren Programmleitung. Die Daten werden vom Schweizerischen Gesundheitsobservatorium ausgewertet. Die Publikation der Meldeergebnisse für 2008 ist auf das 1. Quartal 2010 vorgesehen.

- Umfassende Bedarfs- und Bedürfniserhebung in Zusammenarbeit mit dem kantonsärztlichen Dienst des Kantons Luzern.

- Transkulturelle Anpassung der Informationsbroschüre zur Erkennung und Behandlung von De-

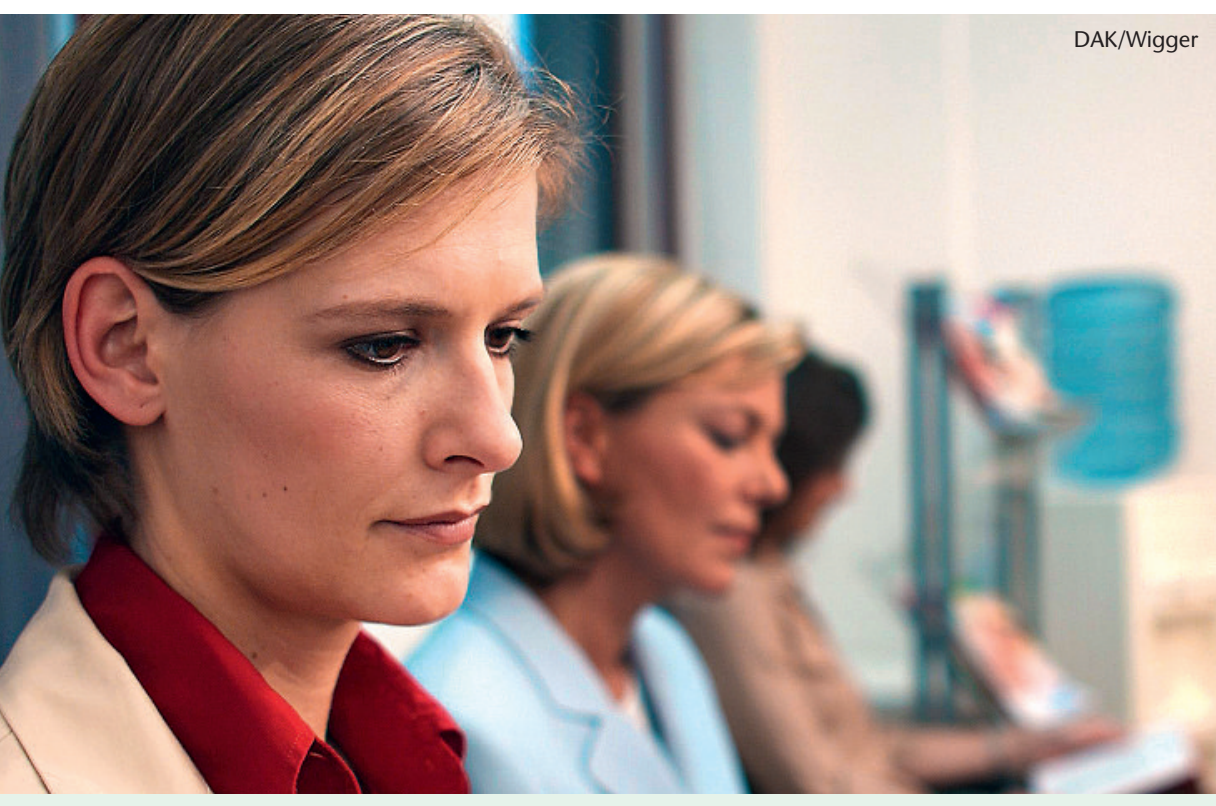

Viele Patienten mit depressiven Erkrankungen gehen zunächst zu ihrem Hausarzt.
Die 3 Botschaften der Informationskampagne «Bündnis gegen Depression»:

- Depression kann jede und jeden treffen.

- Depression hat viele Gesichter.

- Depression ist behandelbar.

pressionen für die Bevölkerung und Übersetzung in die fünf häufigsten Migrationssprachen in der Schweiz im Rahmen der 2. Strategiephase «Migration und Gesundheit».

- Entwicklung von Kompetenzprofilen für die hausärztliche Praxis und weitere Multiplikatoren, durch Fachexperten und im Kontakt mit Fachorganisationen der Ärzteschaft, Psychologie und Pflege, in Anlehnung an die lizenzierten Schulungskonzepte aus dem Deutschen Bündnis gegen Depression.

In Zusammenarbeit mit der Schweizerischen Konferenz der kantonalen Gesundheitsdirektorinnen und -direktoren (GDK) werden für den Wissenstransfer jährliche überregionale Netzwerktreffen durchgeführt. Am Netzwerk beteiligen sich auch Fachorganisationen und interessierte Kantonsvertreter. Das Netzwerk ist auch offen für andere Vorgehensweisen zur Verbesserung der psychischen Gesundheit und zur Reduktion von Suiziden.

\section{Die Depressionskrankheit in der hausärztlichen Praxis}

In der Grundversorgung ist Depression mit einer Prävalenzrate um $10 \%$ vertreten $[7,8]$. Die meisten Patientinnen und Patienten mit Depression konsultieren wegen unspezifischer Beschwerden ihren Hausarzt [9]. Es muss weiter angenommen werden, dass die meisten Suizidanten vor dem Suizid einen Arzt konsultiert haben. Bei $40 \%$ fand eine solche Begegnung sogar in der Woche vor dem Suizid statt [10]. Leider liegen aus der Schweiz keine zuverlässigen Daten vor, doch gehen die Autoren davon aus, dass die erwähnten und auch die folgenden Zahlen aus der internationalen Literatur auch für die Lage in der Schweiz eher zutreffen.

Nach den in diesem Artikel zitierten Studien aus angelsächsischen Ländern, Deutschland, Italien und den Niederlanden scheint bisher die rechtzeitige Erkennung, Behandlung und Verhütung im hausärztlichen Alltag eine Herausforderung zu bleiben: 50-75\% der Depressionen werden in der Grundversorgung dieser Länder nicht erkannt [11-14]. Das Ausmass der Depression wird oftmals unterschätzt [15]. Weil die meisten Studienresultate allerdings auf Einzelkonsultationen beruhen [16-18], die Hausärzte aber typischerweise ihre Patienten mehrmals und über längere Zeit sehen, dürfte dank des Einbezugs der Verlaufserfahrung eine höhere diagnostische Trefferwahrscheinlichkeit erzielt werden. Tatsächlich konnte gezeigt werden, dass initial unerkannte Depressionen häufig innerhalb der nächsten drei Monate doch noch erfasst werden [19]. 
Grundversorger in den USA oder in Deutschland sollen angeblich weniger als jede 3. Person mit einer Depression adäquat behandeln[1, 13, 14]. Sie dosieren Antidepressiva häufig zu tief $[20,21]$ und erreichen damit keine Remission der Depression [21, 22]. In Deutschland sollen nur etwa 10\% der korrekt diagnostizierten Fälle in der Grundversorgung adäquat psychopharmakologisch und/oder psychotherapeutisch behandelt bzw. rechtzeitig an Fachärzte überwiesen werden [1, 13, 14, 19]. Viele Patienten setzen ihre antidepressive Medikation bereits in den ersten drei Monaten ab [23, 24], auch wenn gemäss Europäischen Richtlinien der WHO die Behandlung über sechs Monate fortgeführt werden sollte, um ein unnötiges Rezidiv zu vermeiden (www.euro.who.int/HEN).

Es macht deshalb Sinn, auch in der Schweiz in der Grundversorgung Wissen und Kompetenzen zur Depressionserkennung und -behandlung weiter zu fördern. Neuen wissenschaftlichen Erkenntnissen und Strömungen soll in der Grundversorgung fortlaufend Rechnung getragen werden. Es ist erwiesen, dass die Behandlung einer Depression in der Grundversorgung kostengünstig ist und den Outcome verbessern kann [11], und dass das Erkennen wie die Behandlung der Depression in der Praxis mit entsprechender Sachkenntnis und Vernetzung mit geringem Aufwand verbunden ist. Wir schlagen deshalb zur Förderung von Wissen und Kompetenz die Schaffung eines klar definierten Gegenstandskatalogs zur gezielten Weiter- und Fortbildung der Hausärztinnen und Hausärzte hinsichtlich Depressionserkennung und -behandlung vor, wie er bisher noch nicht existiert.

\section{Entwicklung eines Kompetenzprofils für die hausärztliche Praxis}

Die Erarbeitung von Kompetenzprofilen als Orientierungshilfe für die in der Grundversorgung Tätigen soll die Qualität von Diagnostik, Behandlung und Verhütung der Depression in der Schweiz optimieren. Mit dem in der Schweiz gut ausgebauten und organisierten Aus,- Weiter- und Fortbildungssystem auf nationaler und kantonaler Ebene sollten in Zusammen-
Damit ein solches Kompetenzprofil möglichst viele Ärzte in der Grundversorgung ansprechen kann, sollten bei der Entwicklung folgende Kriterien berücksichtigt werden:

- Unterschiedliche Patientenpopulationen (Geschlecht, Alter, Herkunft)

- Unterschiedliche Komorbiditäten

- Regional unterschiedliche Vernetzungsmöglichkeiten mit Fachärztinnen und -ärzten der Psychiatrie und Psychotherapie

Abhängig von der Praxisstruktur, -ausrichtung und -vernetzung verfügen die meisten Kolleginnen und Kollegen über einen reichen praktischen Erfahrungsschatz hinsichtlich Diagnostik und Therapie. Wichtig und noch zu stärken sind aber Kenntnisse in der Früherkennung und den gängigen psychopharmakologischen und psychotherapeutischen Vorgehensweisen. Eine explorative Befragung einer nicht repräsentativen Stichprobe von Hausärzten und Psychiatern (Begré) lieferte wichtige Hinweise und Ideen zu möglichen Inhalten eines Kompetenzprofils zur unterschiedlichen Machbarkeit bei der Diagnosestellung und Therapie einer Depression und zur Zusammenarbeit zwischen den verschiedenen medizinischen Fachrichtungen hinsichtlich Depressionsverhütung in der hausärztlichen Praxis.

\section{Vorgehensweise}

Die Literaturrecherche, Praxisforschung und die lizenzierten Schulungsmaterialien könnten in ein Kompetenzprofil für die Hausärztinnen und -ärzte münden und zur Verbesserung von Diagnostik und Therapie depressiver Erkrankungen im Sinne einer Orientierungshilfe beitragen, Ein solches Kompetenzprofil könnte dann zur Diskussion unter den Beteiligten gestellt werden. Für jede Phase wird eine Vorgehensweise für die kompetente ärztliche Betreuung depressiv Erkrankter unterschiedlicher Herkunft und Schweregrade definiert. Dabei werden verschiedene Interessenslagen, Ausbildungsstand und Kenntnisse der in der Grundversorgung tätigen Kollegen berücksichtigt.

\section{«Wichtig und noch zu stärken sind Kenntnisse in der Früh- erkennung und den gängigen psychopharmakologischen und psycho- therapeutischen Vorgehensweisen»}

arbeit mit den Fachorganisationen der Ärzteschaft, Pflege und Psychologie nachhaltige Lösungen zur Behandlungs- und Betreuungsoptimierung bei Depression und Suizidalität in der Schweiz erreicht werden. Die Profile sollen auch eine Selbsteinschätzung zu den vorhandenen Kenntnissen und Fertigkeiten bei diesem anspruchsvollen Gesundheitsproblem erleichtern.
Primär kann sich derzeit jeder Arzt anhand der Kenntnisse und Fertigkeiten ausrichten, die in bereits bestehenden Ausbildungsgängen hinsichtlich Depressionsbehandlung vermittelt werden, basierend auf den psychosozialen Lerninhalten, die im Rahmen des FMH-Weiterbildungscurriculums zum Facharzt für Psychiatrie und Psychotherapie oder der Erlangung 
der beiden FMH-Fähigkeitsausweise für psychosoziale Kompetenzen vermittelt werden:

- Kolleginnen und Kollegen ohne ausreichende spezifische Kenntnisse und Fertigkeiten zur Diagnose und Behandlung depressiver Erkrankungen

- Kollegen mit Zusatzausbildung im Sinne des Besitzes eines Fähigkeitsausweises für delegierte Psychotherapie (FA DP) des Dachverbandes der psychiatrisch-psychotherapeutisch tätigen Ärzte und Ärztinnen (FMPP) oder/und eines Fähigkeitsausweises für Psychosomatische und Psychosoziale Medizin (FA SAPPM) oder/und mit mindestens einem Jahr Erfahrung in einer ambulanten und/ oder stationären psychiatrischen Institution während oder nach der Facharztausbildung

- Fortgeschrittene, speziell interessierte Kollegen mit geeigneter spezifischer Therapieausbildung

\section{Finanzierungsmodelle müssen geprüft werden, die Anreize für den Besuch psychosozialer Fortbildungs- angebote mit dem Fokus Depression schaffen könnten}

Es ist uns bewusst, dass eine solche Unterteilung der Zielgruppen eines Kompetenzprofils die tatsächlichen Kenntnisse und Fertigkeiten unter allen Hausärzten nur ungenügend abbildet. Auf der anderen Seite ist sie ja nur als Orientierungshilfe zum jeweiligen Vorgehen in jeder Phase der Depressionsbehandlung gedacht. Ergänzend zum Kompetenzprofil könnten dann in einem dazugehörigen Gegenstandskatalog für Hausärzte die Lerninhalte als Voraussetzung für das Erkennen und Behandeln depressiver Erkrankungen für in der Grundversorgung Tätige systematisch beschrieben werden. Bestehende Weiterbildungs- und Fortbildungsangebote der einzelnen Fach- und regionalen Ärztegesellschaften mit Fokus Depression würden im Gegenstandskatalog integriert, neue Angebote bei Bedarf geschaffen werden. Die Inhalte des Gegenstandskatalogs könnten kontinuierlich den neuesten Erkenntnissen angepasst werden. Unterteilt in essentielle und optionale Inhalte berücksichtigte ein solcher Gegenstandskatalog die unterschiedlichen Bedürfnisse der in der Grundversorgung tätigen Ärzte im Rahmen ihrer Praxisausrichtung. Essentielle Inhalte listeten das absolute Minimum an Kenntnissen und Fertigkeiten für jeden Arzt auf, optional diejenigen für speziell Interessierte, mit Fokus Depression tätige Kollegen. Ein solcher Gegenstandskatalog würde im Sinne der jeweiligen Praxisausrichtung auch eine wertvolle Hilfe bieten für die Zusammenstellung des eigenen Fortbildungscurriculums.

\section{Schlussfolgerungen und Ausblick}

Depression ist weltweit die häufigste Ursache für Erwerbsunfähigkeit und auch in der Schweiz weitver- breitet. Hausärztinnen und -ärzte nehmen bei der Versorgung depressiv Erkrankter eine Schlüsselstellung ein. Es ist erwiesen, dass die Behandlung einer Depression in der Grundversorgung kostengünstig ist und den Outcome verbessern kann. Das Erkennen und die Behandlung einer Depression in der Praxis sind bei entsprechenden Sachkenntnissen und Vernetzung mit geringem Aufwand verbunden, und es macht deshalb Sinn, zusätzliche Massnahmen zur Unterstützung von Diagnosestellung und Therapie in der Grundversorgung zu unternehmen. Eine enge Zusammenarbeit zwischen Hausärzten und Psychiatern ist dabei von grosser Wichtigkeit. Beide Berufsgruppen sind je nach Situation unterschiedlich mit der Versorgung dieser Patientengruppe konfrontiert. Fragestellungen hinsichtlich Diagnosehäufigkeiten, Schweregrad, Komorbiditäten und Therapieverläufen sowie der Vergleich unter den verschiedenen Fachrichtungen sind noch weiter zu bearbeiten. Es existieren in der Schweiz derzeit noch kaum Daten dazu.

Ein besonderes Interesse an psychosozialen Belangen ist eine notwendige Voraussetzung zur Erkennung, Behandlung und Prävention depressiver Störungen. Dieses Interesse sollte bereits in der universitären Ausbildung geweckt werden, und es muss in der postgradualen Weiterbildung zum Facharzt und in der lebenslangen Fortbildung nahtlos weitergehen. Dabei müssen verbindliche Lerninhalte im Sinne eines Gegenstandskataloges zur Diagnostik und Therapie der Depression spezifisch für Hausärzte definiert werden. Das 2007 in Kraft getretene neue Medizinalberufegesetz hat hier klare Rahmenvorgaben gemacht, die im Interesse der Gesundheit von Individuen und Bevölkerung liegen.

Zusatzausbildungen sind allerdings für jede Hausärztin und jeden Hausarzt kostspielig. Verschiedene Finanzierungsmodelle müssen deshalb studiert werden, die für den Besuch psychosozialer Fortbildungsangebote mit Fokus Depression einen Anreiz schaffen könnten. Mit einem entsprechenden Labeling könnten beispielsweise bereits bestehende Fortbildungsangebote durch die ärztlichen Organisationen (insbesondere KHM, SAPPM, SGAM, SGIM, SGPP, SGKJPP, SGP und SGG) als geeignet gekennzeichnet und anhand des definierten Gegenstandskataloges für Depressionsbehandlung in ein Gesamtangebot integriert werden.

Diagnostik und Therapie der Depression bei der Migrationbevölkerung sind aufgrund der sprachlichen und soziokulturellen Barrieren oftmals eine besondere Herausforderung. Eine Liste mit Dolmetschern, Auskunftsstellen sowie speziellen Angeboten für die Behandlung von Patienten aus anderen Kulturkreisen soll den in der Grundversorgung Tätigen zur Verfügung gestellt werden.

Interventionsstudien in Zusammenarbeit mit den Hausärzten stellen eine Möglichkeit dar, um den Effekt von Informationskampagnen der verschiedenen Bündnispartner auf Diagnosestellung und Behand- 
lungsqualität der Depression zu erfassen. Auch die Kostenwirksamkeit einer verbesserten Diagnostik und Therapie müsste überprüft werden. Primäre Ziele solcher Kampagnen sind die Senkung der relativ hohen Suizidrate in der Schweiz und die Verringerung der Krankheitslast.

\section{Literatur}

1 Spiessl H, Hubner-Liebermann B, Schmid R, Cording C, Hajak G. Depressive patients in primary care. MMW Fortschr Med. 2006;148(35-36):42-3.

2 Möller H, Henkel V. What are the most effective diagnostic and therapeutic strategies for the management of depression in specialist care? WHO Regional Office for Europe. Health Evidence Network report. Copenhagen; 2005 www.euro.who.int/Document/E86602.pdf

3 Andrews G. Should depression be managed as a chronic disease? BMJ. 2001. 322(7283):419-21.

4 Hegerl U, Althaus D, Schmidtke A, Niklewski G. The alliance against depression: 2-year evaluation of a community-based intervention to reduce suicidality. Psychol Med. 2006;36(9):1225-33.

5 Frey C. Suizidprävention in der Arztpraxis. Schweiz Ärztezeitung. 1992;73:713-7.

6 Rutz W, Walinder J, Eberhard G, Holmberg G, von Knorring AL, von Knorring $L$ et al. An educational program on depressive disorders for general practitioners on Gotland: background and evaluation. Acta Psychiatr Scand. 1989;79(1):19-26.

7 Pieper L, Schulz H, Klotsche J, Eichler T, Wittchen HU. Depression as a comorbid disorder in primary care. Bundesgesundheitsblatt. Gesundheitsforschung Gesundheitsschutz. 2008;51(4):411-21.

8 Spitzer RL, Williams JB, Kroenke K, Linzer M, de Gruy FV, Hahn SR et al. Utility of a new procedure for diagnosing mental disorders in primary care. The PRIME-MD 1000 study. JAMA. 1994;72(22):1749-56.

9 Wittchen HU, Müller N, Schmidtkunz B, Winter S, Pfister H. Erscheinungsformen, Häufigkeit und Versorgung von Depressionen. Ergebnisse des bundesweiten Gesundheitssurveys «Psychische Störungen». MMW Fortschr Med. 2000;118:4-10.

10 Pirkis J, Burgess P. Suicide and recency of health care contacts. A systematic review. Br J Psychiatry. 1998;173:462-74.

11 Egede LE. Failure to recognize depression in primary care: issues and challenges. J Gen Intern Med. 2007;22(5):701-3.
12 Arroll B, Goodyear-Smith F, Kerse N, Fishman T, Gunn J. Effect of the addition of a «help» question to two screening questions on specificity for diagnosis of depression in general practice: diagnostic validity study. BMJ. 2005;331(7521):884.

13 Bermejo I, Kratz S, Schneider F, Gaebel W, Mulert C, Hegerl U et al. Agreement in physicians' and patients' assessment of depressive disorders. Z Ärztl Fortbild Qualitätssich. 2003;97 Suppl 4:44-9.

14 Jacobi F, Hofler M, Meister W, Wittchen HU. Prevalence, detection and prescribing behavior in depressive syndromes. A German federal family physician study. Nervenarzt. 2002;73(7):651-8.

15 Van Os TW, Van den Brink RH, Van der MK, Ormel J. The care provided by general practitioners for persistent depression. Eur Psychiatry. 2006;21(2):87-92.

16 Simon GE, VonKorff M. Recognition, management, and outcomes of depression in primary care. Arch Fam Med. 1995;4(2):99-105.

17 Pini S, Perkonnig A, Tansella M, Wittchen HU, Psich D. Prevalence and 12-month outcome of threshold and subthreshold mental disorders in primary care. J Affect Disord. 1999;56(1):37-48.

18 Cepoiu M, McCusker J, Cole MG, Sewitch M, Belzile E, Ciampi A. Recognition of depression by non-psychiatric physicians - a systematic literature review and meta-analysis. J Gen Intern Med. 2008;23(1):25-36.

19 Simon GE, Goldberg D, Tiemens BG, Ustun TB. Outcomes of recognized and unrecognized depression in an international primary care study. Gen Hosp Psychiatry. 1999;21(2):97-105.

20 Katon W, von Korff M, Lin E, Bush T, Ormel J. Adequacy and duration of antidepressant treatment in primary care. Med Care. 1992;30(1):67-76.

21 Lin EH, Katon WJ, Simon GE, von Korff M, Bush TM, Walker EA et al. Low-intensity treatment of depression in primary care: is it problematic? Gen Hosp Psychiatry. 2000;22(2):78-83.

22 Weilburg JB, O'Leary KM, Meigs JB, Hennen J, Stafford RS. Evaluation of the adequacy of outpatient antidepressant treatment. Psychiatr Serv. 2003; 54(9):1233-39.

23 Demyttenaere K, Enzlin P, Dewe W, Boulanger B De Bie J, De Troyer et al. Compliance with antidepressants in a primary care setting, 1: Beyond lack of efficacy and adverse events. J Clin Psychiatry. 2001;62 Suppl 22:30-3.

24 Corey-Lisle PK, Nash R, Stang P, Swindle R. Response, partial response, and nonresponse in primary care treatment of depression. Arch Intern Med. 2004;164(11):1197-204. 